\title{
TRANSMISSION SYSTEM EXPANSION PLANNING WITH CONSIDERATION OF N-1 SECURITY CONSTRAINTS
}

\section{Bundhit Eua-arporn and Somphop Asadamongkol}

\author{
Department of Electrical Engineering, \\ Faculty of Engineering, Chulalongkorn University, \\ Bangkok, Thailand 10330
}

\section{ABSTRACT}

This paper presents a heuristic method for solving transmission system expansion problems (TSEP) with consideration of $\mathrm{N}-1$ security constraints. The method is divided into two phases. An initial plan is established in the first phase by a search process which is based on a modified simplex method and sensitivity indices. The second phase starts from the initial plan and performs the local search in the defined neighborhood. Additionally, reconstruction of the new lines on the existing right of ways, which is one of the interesting issues of TSEP in the urban area, is taken into account. The proposed method provides very satisfactory results compared with others.

\section{KEYWORDS}

heuristic method, $\mathrm{N}-1$ security cons traint, simplex method, transmission system expansion planning. 


\section{Introduction}

TRANSMISSION SYSTEM EXPANSION PLANNING (TSEP) is one of the major tasks of an electric utility. With the forecast of electricity demand and the generation expansion plan in the planning horizon, the TSEP concerns with the questions of how to obtain the minimum cost plan which ensures operational security of the power system for delivering energy from generating stations to electricity customers throughout planning horizon [1].

The methods for solving TSEP can be divided into three categories [2], mathematical optimization, heuristic and metaheuristic. From the mathematical point of view, the TSEP is a mixed integer programming and its algorithm complexity is nondeterministic polynomial. In general, the mathematics based methods have to encounter the problem of computation time when it is applied to medium or large scale power systems. In case of taking into account additional constraints, e.g. N-1 security, the computation burden will be more severe. On the other hand, the heuristic based methods, which normally apply a search process guided by specified indices, can provide solution with less computation effort but deem to fall into local optimum. However, with elaborated modification of the search procedure according to the nature of the problem, it is possible to obtain results with acceptable quality. The metaheuristic methods, e.g. genetic algorithm, simulate annealing etc., are similar to the heuristic methods. A key objective of the metaheuristic based method concerns the search process with embedded mechanism to escape from the local optima. Therefore, the methods normally encounter higher computation burden than the heuristic based methods.

In this paper, a heuristic based method for solving the TSEP in single stage planning with $\mathrm{N}$ 1 security constraint consideration is proposed. In addition, the option of the reconstruction of new lines on the existing right of ways is taken into account. The applied search process employs a simplex method and sensitivity indices which reflect the alleviation of system overloaded resulting from construction of new lines.

This paper is composed of 6 sections. Section II reviews the heuristic method for the TSEP. Section III presents a general simplex method. Section IV presents the proposed method. Section $V$ shows the test results of the proposed method. Finally, conclusions are drawn in section VI.

\section{Heuristic Method for TSEP}

Most of the heuristic methods are based on the selection of candidate transmission lines in association with their sensitivity indices which reflect the ability to alleviate overloading problems [3], [4]. In general, it continuously selects a number of new candidate lines so that the system is brought into a safe state. From this point, the set of candidates are established as an initial plan. Then an elimination task is performed by removing a less potential candidate from the initial plan. If the system still resides in a safe state, i.e. no overloaded line, then it can be permanently removed from the plan, otherwise it will be kept in the plan. The elimination task is continuously performed by removing other candidates in such manner until satisfied results are obtained.

In the optimization point of view, the establishment of the initial plan can be compared to the change of system states, i.e. from infeasible into a feasible states, whereas the elimination task can be compared with the local search, which tries to change system states from current to the other having lower cost.

\section{General Simplex Method}

The simplex method is one of the familiar methods for solving a linear programming (LP) problem. Its search process is based on iterative operation. In case of a minimization problem, it tries to change the current feasible solution to the other which has lower value of the objective function. There are various versions of the simplex method which are suitable for implementation in the computer program. However, in this section, the method based on the full tableau, which is useful for describing the proposed method for solving TSEP, is presented. The more details of other methods can be founded in [5].

We begin to describe the simplex method with the LP problem in standard form as follows. 


$\begin{array}{ll}\text { Minimize } & \begin{array}{l}\boldsymbol{z}=\mathbf{c}^{\boldsymbol{\top}} \mathbf{x} \\ \text { Subject to }\end{array} \\ & \mathbf{A x}=\mathbf{b} \\ & \mathbf{x} \geq \mathbf{0}\end{array}$

where $\mathbf{b} \geq \mathbf{0}, \mathbf{c} \in \mathbb{R}^{n}, \mathbf{b} \in \mathbb{R}^{m}, \mathbf{A} \in \mathbb{R}^{m \times n}, m<n$, and rank $\mathbf{A}=m$. A set of feasible points, defined by linear constraints (2) and (3), is sometimes called a polyhedron.

In theory of LP, an optimum solution is one of basic feasible solutions of the problem. Therefore, the simplex method tries to change the current solution, which is one of basic feasible solutions, to the other having lower value of the objective function. From geometric viewpoint, a basic feasible solution and a vertex of polyhedron are one and the same. Additionally, the change of current solution can be compared to the search along edge direction of polyhedron together with the preservation of descent property. The process is performed continuously until there is no descent direction to change the current solution.

Algebraically, if a vector $\mathbf{x}$ is a basic feasible solution, its element can be separated into two subvectors. The first consists of $m$ basic variables, $\mathbf{x}_{\mathbf{B}}$, of which the values are greater than or equal to zeros. Whereas, the second consists of $n-m$ nonbasic variable, $\mathbf{x}_{\mathbf{N}}$, which are all zeros. At this point, we can rearrange the elements of $\mathbf{x}$ as follows.

$$
\mathbf{X}^{\top}=\left[\begin{array}{ll}
\mathbf{X}_{\mathbf{B}}^{\top} & \mathbf{X}_{\mathbf{N}}^{\top}
\end{array}\right]
$$

In addition, the element of $\mathbf{c}$ in objective function (1) and $\mathbf{A}$ in constraints (2) have to be rearrange according to the order of variables in $\mathbf{x}$. Therefore, the LP problem (1)-(3) can be expressed as the following forms.

$$
\begin{array}{ll}
\text { Minimize } & \boldsymbol{Z}=\mathbf{C}_{\mathrm{B}}^{\top} \mathbf{x}_{\mathrm{B}}+\mathbf{C}_{\mathrm{N}}^{\top} \mathbf{x}_{\mathbf{N}} \\
\text { Subject to } & \mathbf{B} \mathbf{x}_{\mathrm{B}}+\mathbf{N} \mathbf{x}_{\mathbf{N}}=\mathbf{b} \\
& {\left[\begin{array}{ll}
\mathbf{X}_{\mathrm{B}}^{\top} & \mathbf{x}_{\mathbf{N}}^{\top}
\end{array}\right]^{\top} \geq \mathbf{0}}
\end{array}
$$

It can be assumed without loss of generality that at the beginning of process, the initial solution, $\mathbf{x}$ is a basic feasible solution, i.e. $\mathbf{x}_{\mathrm{B}} \geq \mathbf{0}$ and $\mathbf{x}_{\mathbf{N}}=\mathbf{0}$. Therefore, the initial state of the tableau, which represents (5), (6), can be represented as.

\begin{tabular}{cccc} 
basic & $\mathbf{X}_{\mathrm{B}}$ & $\mathbf{x}_{\mathrm{N}}$ & $\begin{array}{c}\text { right hand side } \\
\text { (rhs) }\end{array}$ \\
\hline$-z$ & $\mathbf{C}_{\mathrm{B}}^{\top}$ & $\mathbf{C}_{\mathbf{N}}^{\top}$ & 0 \\
\hline $\mathbf{X}_{\mathbf{B}}$ & $\mathbf{B}$ & $\mathbf{N}$ & $\mathbf{b}$ \\
\hline
\end{tabular}

We can derive the relationship between $\mathbf{x}_{\mathbf{B}}$ and $\mathbf{x}_{\mathbf{N}}$ as well as the relationship between $z$ and $\mathbf{x}_{\mathrm{N}}$ from (5), (6) as follows.

$$
\begin{gathered}
\mathbf{X}_{\mathrm{B}}+\mathbf{B}^{-1} \mathbf{N} \mathbf{X}_{\mathbf{N}}=\mathbf{B}^{-1} \mathbf{b} \\
-Z+\left(\mathbf{C}_{\mathbf{N}}^{\top}-\mathbf{C}_{\mathrm{B}}^{\top} \mathbf{B}^{-1} \mathbf{N}\right) \mathbf{X}_{\mathbf{N}}=-\mathbf{C}_{\mathrm{B}}^{\top} \mathbf{B}^{-1} \mathbf{b}
\end{gathered}
$$

Instead of calculating $\mathbf{B}^{-1}$, we can apply the row operation to transform the elements in tableau which correspond to the basic variables, $\mathbf{x}_{\mathbf{B}}$ to $\left[\begin{array}{ll}0 & \mathbf{I}_{m}\end{array}\right]^{\top}$ where $\mathbf{I}_{m}$ is a $m \times m$ identity matrix. Then we can obtain the modified tableau according to (8), (9) as below.

\begin{tabular}{llll} 
basic & $\mathbf{X}_{\mathbf{B}}$ & $\mathbf{X}_{\mathbf{N}}$ & rhs \\
\hline$-Z$ & 0 & $\mathbf{C}_{\mathbf{N}}^{\top}-\mathbf{C}_{\mathrm{B}}^{\top} \mathbf{B}^{-1} \mathbf{N}$ & $-\mathbf{C}_{\mathrm{B}}^{\top} \mathbf{B}^{-1} \mathbf{b}$ \\
\hline $\mathbf{X}_{\mathbf{B}}$ & $\mathbf{I}_{m}$ & $\mathbf{B}^{-1} \mathbf{N}$ & $\mathbf{B}^{-1} \mathbf{b}$ \\
\hline
\end{tabular}


Since $\mathbf{x}_{\mathbf{N}}=\mathbf{0}$, from (9), it can be seen that $z=\mathbf{C}_{B}^{\top} \mathbf{B}^{-1} \mathbf{b}$. In addition, the value of $z$ can be lowered by increasing the value of nonbasic variables to which the negative elements in vector $\mathbf{c}_{\mathbf{N}}^{\top}-\mathbf{C}_{\mathbf{B}}^{\top} \mathbf{B}^{-1} \mathbf{N}$ correspond. Consequently, the $j^{\text {th }}$ element in vector $\mathbf{C}_{\mathbf{N}}^{\top}-\mathbf{c}_{\mathbf{B}}^{\top} \mathbf{B}^{-1} \mathbf{N}, \hat{c}_{j}$ is called the reduced cost of nonbasic variable $x_{j}$. In general, the reduced costs are used in the optimality test to specify whether the current solution is the optimal solution.

The optimality test is performed by examining the value of reduced costs of nonbasic variables. If some of them are less than zeros, the simplex algorithm continues to change the current solution to the other having the lower value of $z$. To achieve this process, it select one of nonbasic variables of which the reduce cost is the most negative value and increase the value of selected variable from zero. The selected variable is called the entering variable, i.e. it will enter to basic variable group. On the other hand, if the reduced costs of all nonbasic variables are greater than or equal to zeros, it means there is no descent direction for changing the current solution. Therefore, the current solution is the optimal solution, then the simplex algorithm is terminated.

The next procedure is concern with the maximum value allowed to increase the entering variable. From (8), it can be seen that the values of basic variables have to be changed when the value of entering variable increase. In addition, the value of entering variable can continuously increase until the value of any basic variable becomes zero. At this point, such basic variable will be changed to nonbasic type, hence it is called the leaving variable, i.e. it will leave from basic variable group. For that reason, the maximum value allowed to increase the entering variable is determined by the value of the leaving variable which becomes zero first.

To obtain the leaving variable, the simplex algorithm performs the ratio test as (10).

$$
i=\underset{1 \leq i \leq m}{\operatorname{argmin}}\left\{\frac{\hat{b}_{i}}{\hat{a}_{i j}}: \hat{a}_{i j}>0\right\}
$$

where $j$ is an index of entering variable, $\hat{b}_{i}$ is an $i^{\text {th }}$ element of vector $\mathbf{B}^{-1} \mathbf{b}$ in the tableau, i.e. rhs column, and $\hat{a}_{i j}$ is an element in $i^{\text {th }}$ row and $j^{\text {th }}$ column of matrix $\left[\begin{array}{lll}\mathbf{I}_{m} & \mathbf{B}^{-1} \mathbf{N}\end{array}\right]$ in the tableau.

After finishing the optimality and ratio test, we will obtain the index of entering variable and leaving variable. In the next step, the pivoting is performed by updating the basic variable group by taking out the leaving variable and bringing in the entering variable, and updating the nonbasic variable group by taking out the entering variable and bringing in the leaving variable. Then the tableau will be updated by applying the row operation to transform the elements which correspond to basic variables to $\left[\begin{array}{ll}0 & \mathbf{I}_{m}\end{array}\right]^{\top}$. Therefore, the reduced costs of nonbasic variables are modified, and all of processes, i.e. optimality test, ratio test and pivoting, are repeated until all the reduced costs are greater than or equal to zeros.

\section{Proposed Heuristic Method}

The proposed method applies an optimization concept. It can be divided into two phases. The first phase deals with the search process based on the best action selection to alleviate overloading problem, which provides an initial plan to be used as a starting point for the local search in the second phase. In general, the search processes in the both phases are similar. However, the process of the second phase will be more elaborated.

\subsection{Overview of Search Process}

In describing the search process, we define the state of the system as

$$
S=(T, \mathbf{g})
$$

where $T$ is a set plans which are subsets of a set of candidate lines $(F)$, and $\mathbf{g} \in \mathbb{R}^{k}$ is the generation schedule for $k$ generators in the system. Moreover, a function, $z_{T}(\mathbf{g})$ which indicates system security is defined as

$$
z_{T}(\mathbf{g})=\sum_{v \in V} z_{T, v}(\mathbf{g}) ; \quad V=E \cup\left\{e_{b}\right\}
$$




$$
z_{T, v}(\mathbf{g})=\sum_{l \in D_{v}}\left(\frac{\left|p_{l, v}(\mathbf{g})\right|}{p_{l, \max }}-1\right) ; \quad D_{v} \subset L
$$

where $E$ is a set of all single outage events, $e_{b}$ is a base case event, i.e. no line outage.

If $E=\varnothing$ the $\mathrm{N}-1$ security constraints are not considered. $D_{v}$ is a set of the overloaded lines for an outage event $v$. $L$ is a set of existing lines in the system. $p_{l, \text { max }}$ is thermal limit of line $l$, and $p_{l, v}(\mathbf{g})$ is the power flow of line $/$ which is an element of vector $\mathbf{p}_{\mathbf{v}}$, expressed as a linear function of $\mathbf{g}$. With a DC model, $\mathbf{p}_{\mathbf{v}}$ can be defined as (14).

$$
\begin{gathered}
\mathbf{p}_{v}=\mathbf{A}_{\mathrm{b}, v} \mathbf{g} \\
\mathbf{A}_{\mathrm{b}, v}=\mathbf{Y}_{\mathrm{pr}, v} \mathrm{CZ}_{\mathrm{bus}, v} \mathbf{K}
\end{gathered}
$$

where $\mathbf{Y}_{\mathbf{p r}, v}$ is a primitive admittance matrix, $\mathbf{C}$ is a branch to bus incidence matrix, $\mathbf{Z}_{\text {bus }, v}$ is a bus impedance matrix and $\mathbf{K}$ is a bus to generator incidence matrix. It should be noted that matrix $\mathbf{Y}_{\mathbf{p r}, v}$ and $\mathbf{Z}_{\text {bus }, v}$ are dependent on the outage event $v$.

The search process tries to move from system state $S$ to the other, resulting in the reduction of $z_{T}(\mathbf{g})$. From (11) it shows that the system state can be changed by two actions. The first one is the construction of new lines, i.e. changing $T$, whereas the second one is the reschedule of the generation, i.e. modifying $\mathbf{g}$.

After adding a few candidate lines to the system, $z_{T}(\mathbf{g})$ may be reduced with modification of $\mathbf{g}$, i.e. generation rescheduling, which can be done by solving the following LP problem.

$$
\begin{aligned}
& \text { Minimize } \\
& \qquad \begin{array}{c}
z_{T}(\mathbf{g}) \\
\text { Subject to } \quad-1 \leq \frac{p_{l, v}(\mathbf{g})}{p_{l, \max }} \leq 1 ; \forall I \in L \backslash D_{v}, \forall v \in V \\
\mathbf{u}_{m}^{\top} \mathbf{g}=d \\
\mathbf{g}_{\min } \leq \mathbf{g} \leq \mathbf{g}_{\max } \\
\mathbf{f}^{\top} \mathbf{g} \leq y_{0}
\end{array}
\end{aligned}
$$

where $\mathbf{u}_{m}$ is a $k \times 1$ matrix of which all elements are ones, $d$ is the total demand, $\mathbf{g}_{\text {min }}$ and $\mathbf{g}_{\max }$ is the operation limits of the generators, $\mathbf{f} \in \mathbb{R}^{k}$ is the vector of the operation cost, and $y_{0}$ is the operation cost of the current state.

In solving the above LP problem, a new developed search algorithm instead of a general simplex method will be applied. We begin to describe that search algorithm with the mathematical formulations which eventually lead to the establishment of the tableau used in the LP solving.

The loading of lines in the system for the event $v$, derived from (14), (15), can be expressed by vector $\mathbf{q}_{v}$ as shown below.

$$
\mathbf{q}_{v}=\mathbf{M} \mathbf{p}_{v}=\left(\mathbf{M} \mathbf{A}_{\mathbf{b}, v}\right) \mathbf{g} ; \forall v \in V
$$

where $\mathbf{M}$ is a diagonal matrix of which the diagonal elements represent the reciprocal of line thermal limits. Additionally, we can formulate the operation constraints of all lines in the system, i.e.

$$
-\mathbf{u}_{n l} \leq \mathbf{q}_{v} \leq \mathbf{u}_{n l} ; \quad \forall v \in V
$$

where $\mathbf{u}_{n l}$ is $n / \times 1$ matrix of which all elements are ones and $n l$ is the number of line in the system. 
From (22) we can obtain the following equations for the lines in the system.

$$
\begin{array}{ll}
\mathbf{q}_{v}+\mathbf{s}_{\mathbf{b}, v}^{+}=\mathbf{u}_{n l} ; & \forall v \in V \\
-\mathbf{q}_{v}+\mathbf{s}_{\mathbf{b}, v}^{-}=\mathbf{u}_{n l} ; & \forall v \in V
\end{array}
$$

where $\mathbf{s}_{b, v}^{+}$and $\mathbf{s}_{\bar{b}, \mathbf{v}}$ are vectors of slack variables. From (21), (23), (24) we can express the set of equations corresponding to the operation constraints of all line in the system for all event $v$ as below.

$$
\mathbf{A}_{\mathrm{gb}} \mathbf{x}_{\mathrm{gb}}=\mathbf{u}_{(2 n / \times n v)}
$$

where $\mathbf{A}_{\mathrm{gb}}$ and $\mathbf{x}_{\mathrm{gb}}$ are defined as

$$
\begin{aligned}
& \mathbf{A}_{\mathrm{gb}}=\left[\begin{array}{ll}
\mathbf{A}_{\mathrm{gb}}^{\mathbf{g}} & \mathbf{I}_{(2 n / \times n v)}
\end{array}\right] \\
& \mathbf{A}_{\mathbf{g b}}^{\mathbf{g}}=\left[\begin{array}{c}
\mathbf{M A}_{\mathbf{b}, 1} \\
-\mathbf{M A}_{\mathbf{b}, 1} \\
\vdots \\
\mathbf{M A}_{\mathbf{b}, n v} \\
-\mathbf{M A}_{\mathbf{b}, n v}
\end{array}\right] \\
& \mathbf{x}_{\mathbf{g b}}^{\top}=\left[\begin{array}{ll}
\mathbf{g}^{\top} & \mathbf{S}_{\mathbf{b}}^{\top}
\end{array}\right] ; \mathbf{S}_{\mathbf{b}}^{\top}=\left[\begin{array}{lllll}
\mathbf{S}_{\mathbf{b}, 1}^{+\top} & \mathbf{S}_{\mathbf{b}, 1}^{-\top} & \ldots & \mathbf{S}_{\mathbf{b}, n v}^{+\top} & \mathbf{S}_{\mathbf{b}, n v}^{-\top}
\end{array}\right]
\end{aligned}
$$

where $n v$ is number of events in $V$.

In addition, constraints (19), (20) can be transformed to a standard form, according to the simplex algorithm, as shown in (29)-(31).

$$
\begin{array}{ll}
\mathbf{g}+\mathbf{s}_{\mathbf{g}}=\mathbf{g}_{\max } ; & \mathbf{s}_{\mathbf{g}} \geq \mathbf{0} \\
\mathbf{g}-\mathbf{e}_{\mathbf{g}}=\mathbf{g}_{\min } ; & \mathbf{e}_{\mathbf{g}} \geq \mathbf{0} \\
\mathbf{f}^{\top} \mathbf{g}+\boldsymbol{s}_{c}=y_{0} ; & \boldsymbol{s}_{c} \geq 0
\end{array}
$$

where $\mathbf{s}_{\mathrm{g}}, \mathbf{e}_{\mathrm{g}}$ are the vector of slack and excess variables of constraint (19) and $s_{c}$ is a slack variable of constraint (20).

From definition of $z_{T}(\mathbf{g})$ in (12), (13), it should be noted that the objective function (16) and the constraints (17) are dynamic. Since the generation schedules have to be modified in during each iteration. Consequently, the lines which are members of $D_{v}$ in the current iteration may not be members of $D_{v}$ in the next iteration. At this point, it can be seen that the lines in the system are divided into two groups. The first group comprises the lines which are members of $D_{v}$ and used in the calculation of $z_{T}(\mathbf{g})$. The second group is composed of the lines which are not the member of $D_{v}$ and will be treated according to (17).

Since we can not permanently specify which lines are involved in the calculation of objective function (16), and also which lines are involved in the calculation of constraint (17). For this reason the tableau of new developed algorithm have to be composed of the operation constraints of all lines in the system.

By combining (25), (29), (30), (31), we can reformulate the set of equations as

$$
\mathbf{A x}=\mathbf{b}
$$




$$
\begin{aligned}
\mathbf{A} & =\left[\begin{array}{ccccc}
\mathbf{A}_{\mathbf{g b}}^{\mathbf{g}} & \mathbf{I}_{2 n / \times n v} & \mathbf{0} & \mathbf{0} & \mathbf{0} \\
\mathbf{I}_{k} & \mathbf{0} & \mathbf{I}_{k} & \mathbf{0} & \mathbf{0} \\
\mathbf{I}_{k} & \mathbf{0} & \mathbf{0} & -\mathbf{I}_{k} & \mathbf{0} \\
\mathbf{f}^{\top} & \mathbf{0} & \mathbf{0} & \mathbf{0} & 1 \\
\mathbf{u}_{k}^{\top} & \mathbf{0} & \mathbf{0} & \mathbf{0} & 0
\end{array}\right] \\
\mathbf{b}^{\top} & =\left[\begin{array}{lllll}
\mathbf{u}_{(2 n \mid \times n v)}^{\top} & \mathbf{g}_{\max }^{\top} & \mathbf{g}_{\min }^{\top} & y_{0} & d
\end{array}\right] \\
\mathbf{x}^{\top} & =\left[\begin{array}{lllll}
\mathbf{g}^{\top} & \mathbf{s}_{\mathbf{b}}^{\top} & \mathbf{s}_{\mathbf{g}}^{\top} & \mathbf{e}_{\mathbf{b}}^{\top} & s_{c}
\end{array}\right]
\end{aligned}
$$

Now we can establish the tableau according to (32), (33), (34), (35) as follows.

\begin{tabular}{lllllll} 
Variable & $\mathbf{g}$ & $\mathbf{s}_{\mathbf{b}}$ & $\mathbf{s}_{\mathbf{g}}$ & $\mathbf{e}_{\mathbf{g}}$ & $\mathbf{s}_{\mathrm{c}}$ & rhs \\
\hline Line loading & $\mathbf{A}_{\mathbf{g b}}^{\mathrm{g}}$ & $\mathbf{I}_{(2 n / \times n v)}$ & $\mathbf{0}$ & $\mathbf{0}$ & $\mathbf{0}$ & $\mathbf{u}_{(2 n \mid \times n v)}$ \\
\hline Max. of $\mathbf{g}$ & $\mathbf{I}_{k}$ & $\mathbf{0}$ & $\mathbf{I}_{k}$ & $\mathbf{0}$ & $\mathbf{0}$ & $\mathbf{g}_{\max }$ \\
\hline Min. of $\mathbf{g}$ & $\mathbf{I}_{k}$ & $\mathbf{0}$ & $\mathbf{0}$ & $-\mathbf{I}_{k}$ & $\mathbf{0}$ & $\mathbf{g}_{\min }$ \\
\hline Cost & $\mathbf{f}^{\top}$ & $\mathbf{0}$ & $\mathbf{0}$ & $\mathbf{0}$ & $\mathbf{1}$ & $\boldsymbol{y}_{\mathbf{0}}$ \\
\hline Demand & $\mathbf{u}_{k}^{\mathrm{T}}$ & $\mathbf{0}$ & $\mathbf{0}$ & $\mathbf{0}$ & $\mathbf{0}$ & $\boldsymbol{d}$ \\
\hline
\end{tabular}

From above tableau, it can be seen that there are $3 k+2 n l \times n v+1$ variables and $2 k+2 n l \times n v+2$ equations. Therefore, the number of basic variable is $2 k+2 n l \times n v+2$, while that of nonbasic variable is $k-1$.

It should be noted that we can use the value of $\mathbf{s}_{\mathrm{b}}$ 's elements to indicate the overloaded lines in the system, e.g. a negative elements indicate the corresponding lines are overloaded. In the new developed algorithm, the elements of $\mathbf{s}_{\mathrm{b}}$ can be any value, while those of $\mathbf{g}, \mathbf{s}_{\mathbf{g}}, \mathbf{e}_{\mathbf{g}}$ and $s_{c}$ have to be greater than or equal to zeros.

It can be assumed without loss of generality that the initial solution, $\mathbf{x}$ is a basic feasible solution, i.e. $x_{i}=0$ if and only if $i \in N$, and $x_{i} \geq 0$ if $i \in B \backslash X_{\mathrm{sb}}$ where $B$ is an index set of basic variables, $N$ is an index set of nonbasic variables and $X_{\mathrm{sb}}$ is an index set of variables which are originally in $\mathbf{s}_{\mathbf{b}}$. By rearranging the elements in matrix $\mathbf{A}$ according to the order of variables in $\mathbf{x}_{\mathbf{B}}$ and $\mathbf{x}_{\mathbf{N}}$, the initial tableau can be described as follows.

\begin{tabular}{llll} 
basic & $\mathbf{x}_{\mathrm{B}}$ & $\mathbf{x}_{\mathbf{N}}$ & rhs \\
\hline $\mathbf{x}_{\mathbf{B}}$ & $\mathbf{B}$ & $\mathbf{N}$ & $\mathbf{b}$ \\
\hline
\end{tabular}

By applying the row operation to transform elements which correspond to the basic variables, $\mathbf{x}_{\mathbf{B}}$ in the tableau to $\mathbf{I}$, we can obtain the modified tableau as follows.

\begin{tabular}{llll} 
basic & $\mathbf{X}_{\mathbf{B}}$ & $\mathbf{X}_{\mathbf{N}}$ & rhs \\
\hline $\mathbf{X}_{\mathbf{B}}$ & $\mathbf{I}$ & $\mathbf{B}^{-1} \mathbf{N}$ & $\mathbf{B}^{-1} \mathbf{b}$ \\
\hline
\end{tabular}

Now we can define a set of overloaded line for all the events in $V$ as

$$
D=\left\{i: i \in B \cap X_{s b} \wedge \hat{b}_{i}<0\right\}
$$

where $\hat{b}_{i}$ is the $i^{\text {th }}$ element of $\mathbf{B}^{-1} \mathbf{b}$, i.e. rhs column of the tableau.

Consequently, we can calculate $z_{T}(\mathbf{g})$ by

$$
z_{T}(\mathbf{g})=-\sum_{i \in D} \hat{b}_{i}
$$


As previously described, if $j$ is a member of $X_{\mathrm{sb}}, x_{j}$ can be a negative value. Therefore, if $x_{j}$ is of nonbasic type, it can be changed in either positive or negative direction from zero. At this point, we will divide the nonbasic variables into two groups depending on their changing direction.

The first group comprises the nonbasic variables which are not the member of $X_{\mathrm{sb}}$, i.e. originated from $\mathbf{g}, \mathbf{s}_{\mathbf{g}}, \mathbf{e}_{\mathbf{g}}$ and $s_{c}$. Therefore, the changes of their values are allowed only in the positive direction. The second group consists of the nonbasic variables which are members of $X_{\mathrm{sb}}$. The changes of their values are allowed in either positive or negative direction.

At this time, we will define new parameters, i.e. $d_{j+}$ and $d_{j-}$, which are similar to the reduced cost of nonbasic variable in general simplex method. However in the new developed algorithm, $d_{j+}$ signifies the reduction of $z_{T}(\mathbf{g})$ when nonbasic variable $x_{j}$ increase, while $d_{j-}$ signifies the reduction of $z_{T}(\mathbf{g})$ when nonbasic variable $x_{j}$ decrease.

It can be noticed that the element in th $i^{\text {th }}$ row and $j^{\text {th }}$ column of matrix $\left[\begin{array}{ll}\mathbf{I} & \mathbf{B}^{-1} \mathbf{N}\end{array}\right]$, where $i \in D$, has impact on the change of $z_{T}(\mathbf{g})$ when $x_{j}$ increases. Additionally, if there exist a zero element $k$ in $\mathbf{B}^{-1} \mathbf{b}$, for $k \in X_{\mathrm{sb}}$, i.e. $x_{k}$ is zero, and in such row $k$ of $\left[\mathbf{I} \mathbf{B}^{-1} \mathbf{N}\right.$ ] there exist a positive element $j$, then $x_{k}$ will be decreased to negative value when $x_{j}$ increase. The decrease of $x_{k}$ means the line corresponding to $k^{\text {th }}$ row is becoming overloaded. Therefore, in the calculation of of $d_{j+}$, that line should be taken into account. From the reason described above, we can define $d_{j+}$ as follows.

$$
\begin{aligned}
& d_{j+}=\sum_{i \in D} \hat{a}_{i j}+\sum_{k \in M^{j+}} \hat{a}_{k j} ; j \in N \\
& M^{j+}=\left\{k: \hat{b}_{k}=0 \wedge \hat{a}_{k j}>0\right\}
\end{aligned}
$$

where $\hat{a}_{i j}$ is element in th $i^{\text {th }}$ row and $j^{\text {th }}$ column of $\left[\mathbf{I} \mathbf{B}^{-1} \mathbf{N}\right]$ and $\hat{b}_{k}$ is $k^{\text {th }}$ element in $\mathbf{B}^{-1} \mathbf{b}$. In the calculation of $d_{j-}$, we can apply the same concept as that of $d_{j+\cdot}$. However, with the decreased value of the nonbasic variable $x_{j}$, the line which is corresponds to $x_{j}$ will become overloaded. Therefore, in the formulation of $d_{j-}$, such overloaded line has to be taken into account, i.e.

$$
\begin{aligned}
& d_{j-}=-\sum_{i \in D} \hat{a}_{i j}-\sum_{k \in M^{j+}} \hat{a}_{k j}+1 ; \quad j \in X_{\mathrm{sb}} \\
& M^{j-}=\left\{k: \hat{b}_{k}=0 \wedge \hat{a}_{k j}<0\right\}
\end{aligned}
$$

After the calculation of $z_{T}(\mathbf{g})$ and reduced costs of nonbasic variables, i.e. $d_{j+}, \forall j \in N$ and $d_{j-}, \forall j \in X_{\text {sb }}$, the optimality test of the new developed algorithm, which is similar to that of the general simplex method, are performed by selecting a nonbasic variable of which the reduced cost is the most negative value as (42).

$$
j=\underset{j \in N}{\operatorname{argmin}}\left\{d_{j+}: d_{j+}<0, \forall j \in N\right\} \cup\left\{d_{j-}: d_{j-}<0, \forall j \in X_{\mathrm{sb}}\right\}
$$

The selected nonbasic variable, $x_{j}$ is called entering variable, and its change direction is determined by whether $d_{j+}$ or $d_{j-}$ is the minimum reduced cost. For the example, if $d_{t+}$ is the minimum reduced cost, the nonbasic variable $x_{t}$ will be selected as the entering variable, and it will be changed in the positive direction, i.e. increase of the value. On the other hand, if $d_{t-}$ is the minimum reduced cost, the nonbasic variable $x_{t}$ will be selected as the entering variable, and it will be changed in the negative direction.

After obtaining the index of entering variable and its changing direction, the ratio test will be performed to determine step size of the change, i.e. the maximum value allowed to change the entering variable. As in the general simplex method, we never obtain the step size explicitly, instead we will obtain index of the leaving variable, i.e. the basic variable of which the value is becoming zeros first when the entering variable is constantly changing. 
From (8), it can be seen that when the value of a nonbasic variable, $x_{j}$ increases, the value of basic variable, $x_{i}$ may be changed depending on the value of $\hat{a}_{i j}$. If $\hat{a}_{i j}$ is a positive number, $x_{i}$ will be decreased. On the other hand, if $\hat{a}_{i j}$ is a negative number, $x_{i}$ will be increased. In addition, if $\hat{a}_{i j}$ is zero, $x_{i}$ will remain unchange.

Therefore, in the new developed algorithm, when the value of entering variable $x_{j}$ increase, the leaving variable is determined by

$$
i=\underset{i \in B}{\operatorname{argmin}}\left\{t_{i}=\frac{\hat{b}_{i}}{\hat{a}_{i j}}: t_{i}>0 ; \hat{a}_{i j} \neq 0\right\}
$$

It should be noted that, in the general simplex method, only basic variables corresponding to the positive $\hat{a}_{i j}$ are examined in the ratio test. However, in this new developed algorithm, the basic variables of which the ratios of $\hat{b}_{i}$ to $\hat{a}_{i j}$ are greater than zeros are examined. The reason is that in the new developed algorithm, the values of $\hat{b}_{i}$ can be less than zeros. Therefore, they have to be tested also in case that their values are increased and reach zeros.

On the contrary, if the nonbasic variable, $x_{j}$ decrease. The leaving variable can be determined by the ratio test which is derived from the same concept as the ratio test in case that $x_{j}$ increases, i.e.

$$
i=\underset{i \in B}{\operatorname{argmin}}\left\{t_{i}=\frac{\hat{b}_{i}}{\hat{a}_{i j}}: t_{i}<0 ; \hat{a}_{i j} \neq 0\right\}
$$

Once the leaving variable is obtained, the pivoting, similar to the general simplex method, is performed by updating the index set of basic variables, $B$, and the index set of nonbasic variables, $N$. Moreover, the tableau will be updated by applying the row operation to transform the elements corresponding to basic variables to [ $\left[\begin{array}{ll}0 & \mathbf{I}_{m}\end{array}\right]^{\top}$. Therefore, all elements in the tableau will be modified. The whole processes, i.e. optimality test, ratio test and pivoting, are repeated until all reduced costs are greater than or equal to zero. Moreover, the information obtained from the last iteration in the tableau after the termination of LP solving can be used for the decision in the following step of the search process.

After solving the above LP problem with the developed algorithm, we will obtain the modified generation schedule, $\mathbf{g}^{*}$. If the overloading problems still exist, i.e. $D \neq \varnothing$, it can be concluded that there is no reduction of $z_{T}(\mathbf{g})$ together with the decrease in the operation cost in either direction. Therefore, the next calculation step is to calculate two types of indices, i.e. generation rescheduling with the increase of the cost $\left(I_{G}\right)$, and the construction of the candidate lines $\left(I_{T, I+}\right)$.

The meaning of $I_{G}$ reflects the decremental cost of $z_{T}(\mathbf{g})$ when the generation cost is increase. In a viewpoint of the simplex method, it can be compared with the change of the system state in the direction which reduces $z_{T}(\mathbf{g})$ and decreases the value of slack variable of (20). Additionally, there are two condition of constraint (20) after solving the LP problem.

Firstly, the constraint is active, i.e. its slack variable, $s_{c}$ is nonbasic type. Since the reduced cost of all nonbasic variable is always greater than zero after terminating the LP solving process. Therefore, the decrease of $s_{c}$ will result in the reduction of $z_{T}(\mathbf{g})$ and the increase of the generation cost to be greater than $y_{0}$. Consequently, $I_{G}$ can be defined to be equal to the reduced cost of the slack variable $s_{c}$.

Secondly, if the constraint (20) is not active, $s_{c}$ is not zero. Therefore, the current value of generation cost is less than $y_{0}$. This condition mean that $s_{c}$ can not be changed in either positive or negative direction to reduce $z_{T}(\mathbf{g})$, i.e. the generator can not reschedule to alleviate the overloading problem. Therefore, in this condition $I_{G}$ will be undefined, and it will not be included in the process of index selection.

The other index, $I_{T, I+}$ represents the decremental cost of $z_{T}(\mathbf{g})$ due to construction of the candidate lines, which can be described as follows: 


$$
I_{T, I+}=\frac{Z_{T}(\mathbf{g})-Z_{T, I+}(\mathbf{g})}{C_{l}}
$$

where $z_{T, I+}(\mathbf{g})$ is defined as $z_{T}(\mathbf{g})$ in (2) but with existency of line $/$ in the system, $c_{l}$ is the annualized construction cost of line $I$. It should be noted that if the line $I$ is constructed on the existing right of way, the removing of the existing line should be taken into account in the calculation of $z_{T, I+}(\mathbf{g})$.

The technique of current injection [6] can be applied to calculate the changed currents of all the lines in the system after adding line $I$.

\subsection{Establishment of Initial plan (The First Phase)}

The objective of this process is to find an initial plan which is in the feasible region. The procedure begins with the search process by solving the LP problem, resulting in $S^{*}=\left(T, \mathbf{g}^{*}\right)$, indices $I_{G}$ and $I_{T, I_{+}}$of candidate lines. If some lines are still overloaded, the process will select actions, i.e. either rescheduling generation or construction of candidate lines, which corresponds to the maximum index. Therefore, if $I_{G}$ is selected, the next action is to reschedule the generation by applying the ratio test in (44) to obtain the leaving variable. In this case, the entering variable is $s_{c}$ and it will be changed in the negative direction. After finishing the ratio test, the pivoting will be performed.

On the other hand if an $I_{T, l+}$ is selected, the change of $T$ may occur, i.e. adding the corresponding line to the system, resulting in the change in matrix $\mathbf{Y}_{\mathrm{pr}, v}, \mathbf{C}$ and $\mathbf{Z}_{\text {bus }, v}$ and all of relevant equations in the LP have to be updated for the next action. The search process continuously proceeds until there is no overloaded line in the system. Finally, it returns an initial feasible plan. A flowchart of the search process is shown in Figure. 1.

\subsection{Local Search (The Second Phase)}

Due to nonconvex nature of the TSEP, it is rarely to find an initial plan as the global optimum solution. Therefore, in the second phase, the algorithm applies the local search in the neighborhood of the initial plan. The concept of the second phase is to disturb the current state of $T$ to move to the other one which has relatively lower cost. If the changing state is infeasible, it will search for a new one which is feasible with lower cost. The main algorithm of the second phase is described below.

1). For the current plan, $S=(T, \mathbf{g})$, set $n i=0$ and $C_{0}=\operatorname{Cost}(S)$.

2). Define $R \subset T$, which comprises $n r$ lines ranked in the top highest values of the construction cost.

3). Set $S^{\prime}=(T \backslash R, \mathbf{g})$. Call LocalSearch $\left(S^{\prime}, C_{0}\right)$ to obtain result $\left(S_{\text {new }}\right.$, success).

4). If success is TRUE then set $S=S_{\text {new }}$ and go to 1), otherwise go to 5).

5). Set $n i=n i+1$. If $n i>$ maxtries, terminate with $S$, otherwise define $R \subset T$ in the same manner as 2 ) but the combination of the element in $R$ is not same as the previous ones, and go to 3 ).

The flowchart of the main algorithm of the second phase is shown in Fig. 2. 
Figure 1

Flowchart of the first phase.

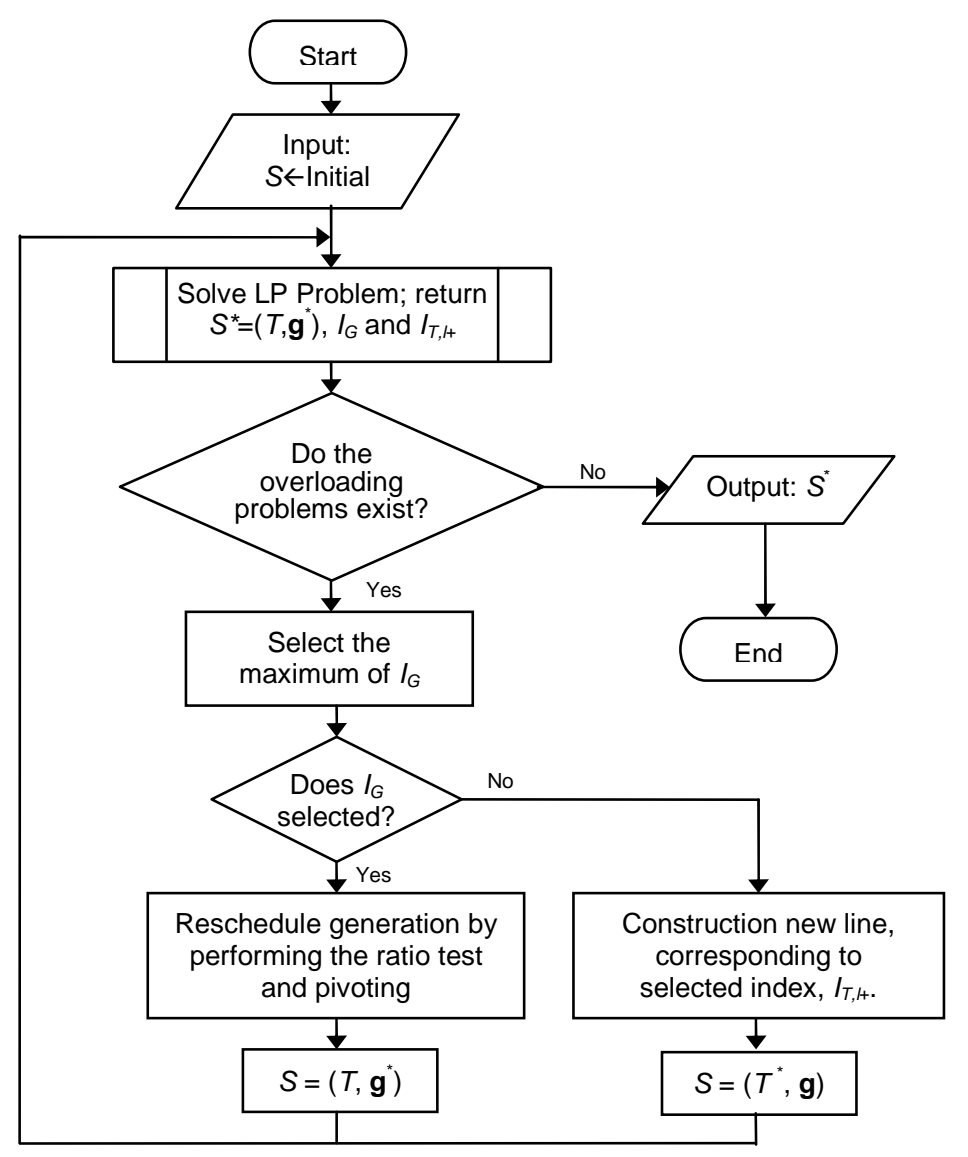

Figure 2

Main algorithm of the second phase.

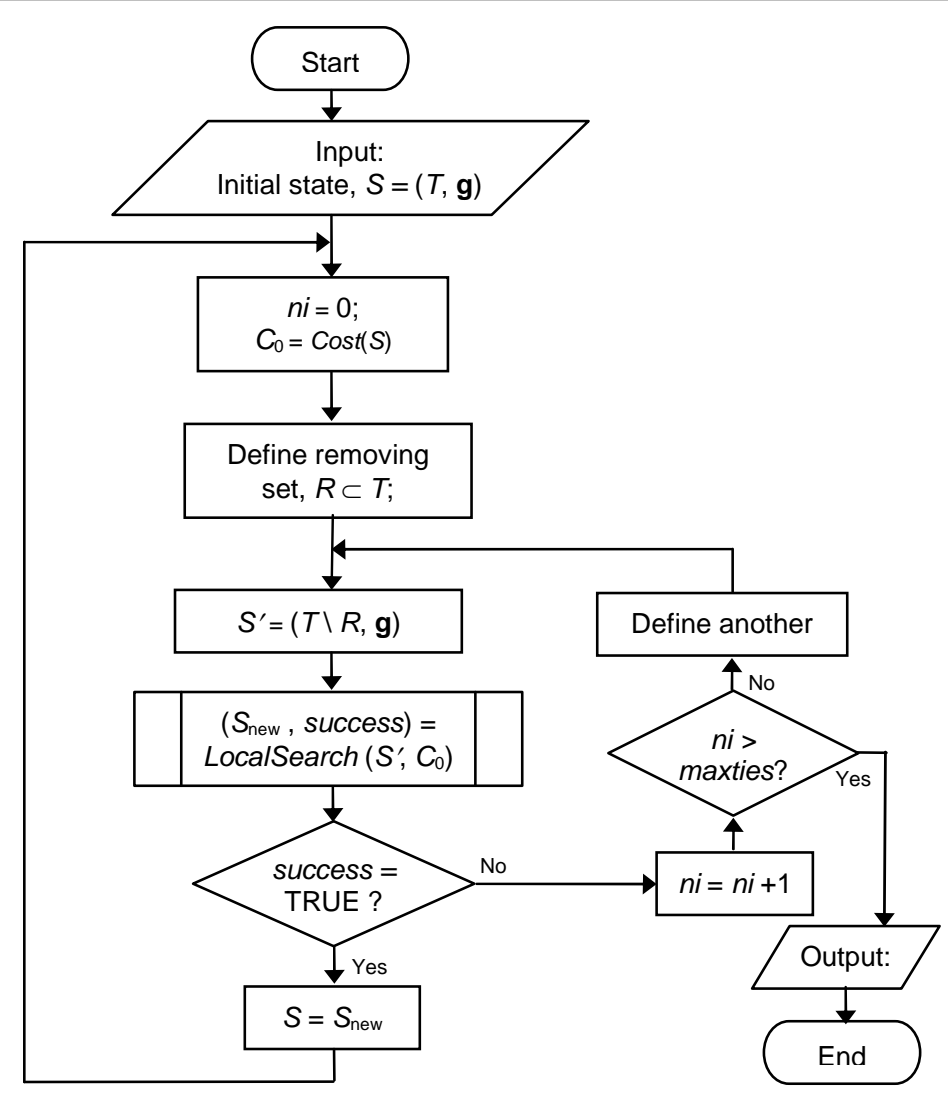




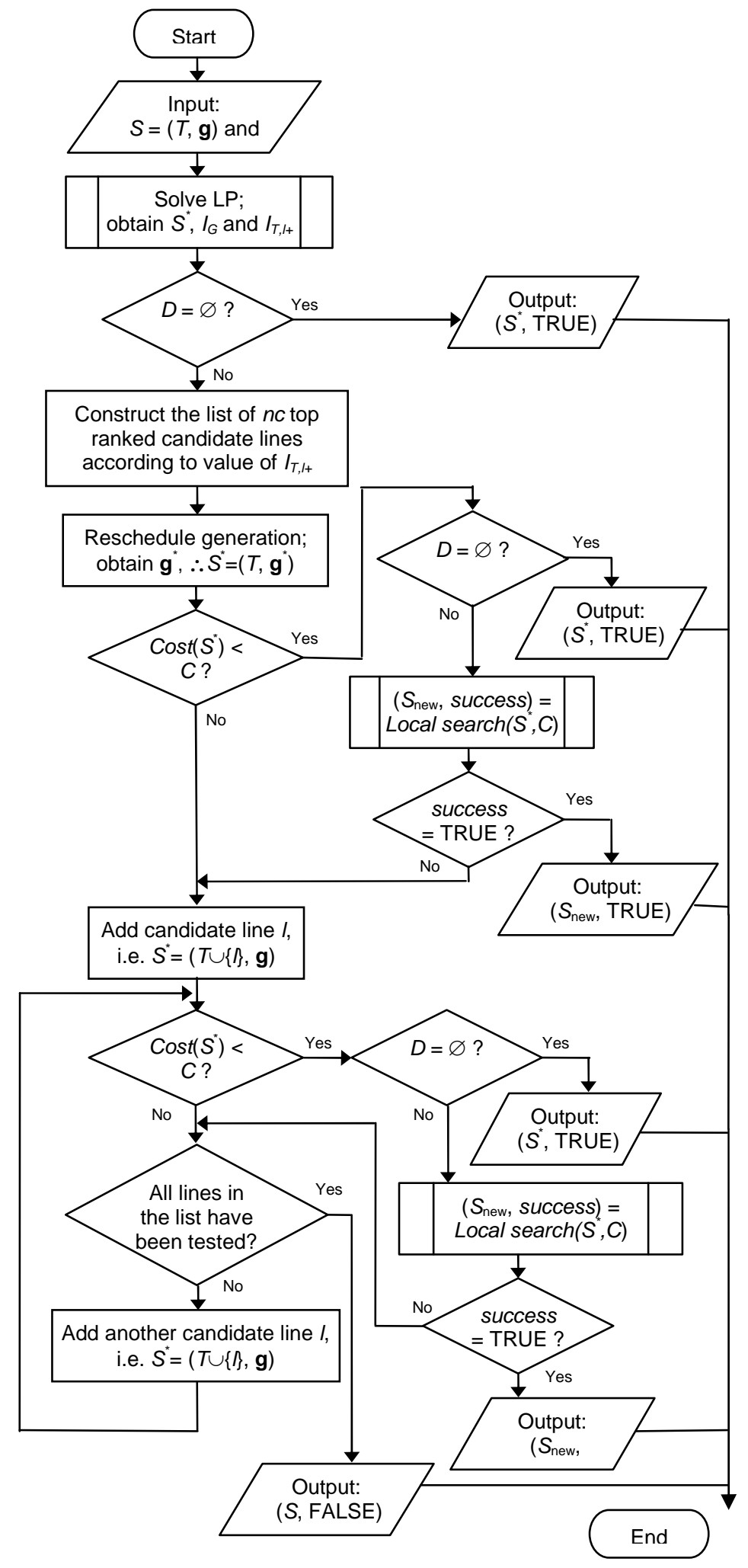

The function $\operatorname{Cost}(S)$ in 1) returns the summation of construction cost of plan $T$ and generation cost due to $\mathbf{g}$. In addition, the subroutine LocalSearch $(S, C)$ in 3 ) will return the success variable with TRUE if it can find $S_{\text {new }}$ with $\operatorname{Cost}\left(S_{\text {new }}\right)<C$. The algorithm is developed based on a recursive procedure described below.

1) From the input $S=(T, \mathbf{g})$ and $C$. Apply the search process by solving LP problem. Obtain state $S^{*}=\left(T, \mathbf{g}^{*}\right)$, indices $I_{G}$ and $I_{T, l_{+}}$of the candidate lines. If the system is feasible, i.e. $D=\varnothing$, terminate with $\left(S^{*}\right.$, TRUE), otherwise construct the list of $k$ top ranked candidate lines according to the values of $I_{T, \mid+}$. 
2) There are one action for adjusting of $\mathbf{g}$, and $n c$ actions for adjusting of $T$. Begin with adjusting $\mathbf{g}$ by the row operation as in a general simplex method. The leaving variable is determined by (44). Obtain result $S^{*}=\left(T, \mathbf{g}^{*}\right)$. If $\operatorname{Cost}\left(S^{*}\right)<C$, perform the next test in 3), otherwise go to 4 ).

3) If the state $S^{*}$ is feasible, terminate with $\left(S^{*}\right.$, TRUE), otherwise ( $S_{\text {new }}$, success $)=$ LocalSearch $\left(S^{*}, C\right)$. If success is TRUE, terminate with $\left(S_{\text {new, }}\right.$, TRUE), otherwise go to 4).

4) Add a candidate line $/$ from the list to $T$, result in $S^{*}=(T \cup\{\}, \mathbf{g})$. If $\operatorname{Cost}\left(S^{*}\right)<C$, perform the next test in 5), otherwise add the next line $/$ from the list to $T$. If all candidate lines have been tested, terminate with ( $S$, FALSE).

5) If the state $S^{*}$ is feasible, terminate with $\left(S^{*}\right.$, TRUE), otherwise ( $S_{\text {new }}$, success $)=$ LocalSearch $\left(S^{*}, C\right)$. If success is TRUE, terminate with $\left(S_{\text {new, }}\right.$, TRUE), otherwise go to 4).

The flowchart of the local search procedure is shown in Figure. 3.

\section{Test Results}

In this section, the proposed method has been tested on a common test system in TSEP research [6]. It is a six bus system with $760 \mathrm{MW}$ of the total demand and the installed capacity is $1110 \mathrm{MW}$. Detailed information can be founded in [3] and [7].

The tests are conducted by two cases, 1) TSEP with $\mathrm{N}-1$ security constraints and 2) TSEP with $\mathrm{N}-1$ security constraints in the situation of the deficiency of the right of way. In both case, there are two options. The first one minimize the total of investment and operation cost, while the second one only minimize the investment cost. The value of $n r$ and maxtries in the main algorithm are defined as 2 and 25 respectively, while the value of $n c$ the local search procedure is 3 .

\subsection{TSEP with N-1 Security Constraints.}

The obtained results are shown in Table I. The results show that although investment cost of the second plan $(180,000$ US $\$ / y r)$ is less than that of the first plan $(200,000$ $\mathrm{U} \$ / \mathrm{yr}$ ), but the operation cost of the first plan is lower. Therefore, in view of the total cost, the first plan provides the total minimum cost plan.

By applying the mathematics optimization method as stated in [7] and [8], we can obtain the same result as the proposed method. However, the computation time on the same computer is about 264.2 and 91.4 seconds respectively.

In [8], the genetic algorithm is applied and only investment cost is minimized. By comparison the results show the second plan provides the same results as [8].

\subsection{TSEP with N-1 Security Constraints and Deficiency of ROW}

The proposed method has been tested in the case that there is only one right of way for each bus pair, which reflects actual situation. In addition, the system planner can decide to construct towers which have more than one circuit on each right of way.

With the addition of the practical standard types, e.g. double circuit and four circuit towers of candidate lines, the construction cost is assumed to be 1.5 and 2.5 times of a single circuit tower respectively. The results are shown in Table II. It should be noted that the line on path 3-5 is reconstructed from the single circuit tower to the four circuit tower on the existing right of way. 


\begin{tabular}{|c|c|c|c|c|}
\hline Path & $\begin{array}{c}\text { Number of } \\
\text { circuit }\end{array}$ & $\begin{array}{c}\text { Investment cost } \\
\left(10^{3} \mathrm{US} \$ / \mathrm{yr}\right)\end{array}$ & $\begin{array}{c}\text { Operation cost } \\
\left(10^{3} \mathrm{US} \$ / \mathrm{yr}\right)\end{array}$ & $\begin{array}{c}\text { Total cost } \\
\left(10^{3} \mathrm{US} \$ / \mathrm{yr}\right)\end{array}$ \\
\hline
\end{tabular}

The first plan: Minimize the total cost

\begin{tabular}{|l|l|l|l|l|}
\hline $2-3$ & 2 & 40 & & \\
\hline $2-6$ & 1 & 30 & \multirow{2}{*}{141,912} & \multirow{2}{*}{142,112} \\
\hline $3-5$ & 2 & 40 & & \\
\hline $4-6$ & 3 & 90 & & \\
\hline
\end{tabular}

The second plan: Minimize the investment cost

\begin{tabular}{|l|l|l|l|l|}
\hline $2-3$ & 1 & 20 & & \\
\hline $2-6$ & 1 & 30 & \multirow{2}{*}{144,467} & \multirow{2}{*}{144,647} \\
\hline $3-5$ & 2 & 40 & & \\
\hline $4-6$ & 3 & 90 & & \\
\hline
\end{tabular}

Table 1

The Plans with $\mathrm{N}-1$ Security Constraints

\begin{tabular}{|c|c|c|c|c|}
\hline Path & $\begin{array}{c}\text { Number of } \\
\text { circuit }\end{array}$ & $\begin{array}{c}\text { Investment cost } \\
\left(10^{3} \text { US } \$ / y r\right)\end{array}$ & $\begin{array}{c}\text { Operation cost } \\
\left.10^{3} \mathrm{US} \$ / \mathrm{yr}\right)\end{array}$ & $\begin{array}{c}\text { Total cost } \\
\left(10^{3} \text { US } \$ / y r\right)\end{array}$ \\
\hline \multicolumn{5}{|c|}{ The first plan: Minimize the total cost } \\
\hline $2-6$ & 2 & 45 & \multirow{4}{*}{141,912} & \multirow{4}{*}{142,111} \\
\hline $3-4$ & 1 & 59 & & \\
\hline $3-5$ & 4 & 50 & & \\
\hline $4-6$ & 2 & 45 & & \\
\hline \multicolumn{5}{|c|}{ The second plan: Minimize the investment cost } \\
\hline $2-6$ & 4 & 75 & \multirow{3}{*}{145,225} & \multirow{3}{*}{145,395} \\
\hline $3-5$ & 4 & 50 & & \\
\hline $4-6$ & 2 & 45 & & \\
\hline
\end{tabular}

\section{Conclusion}

A heuristic based method to solve the transmission system expansion planning with $\mathrm{N}-1$ security constraints has been developed. The search process is based on indices of the actions to remedy the transmission line overloaded problem. In addition the simplex method has been adopted by slight modification to accommodate for the TSEP.

The process of the local search is a critical issue in the developed algorithm. The value of $n r$ can be compared with the size of the neighborhood. The higher value of $n r$ represents the further move from the current plan, which requires longer computation time. In addition, the value of $n c$ and maxtries can be compared with fineness of the search process in the neighborhood. Certainly, the higher value of $n r, n c$ and maxtries can provide a better final 
plan, however it may cause higher computation burden. Therefore, the compromise between the quality of final plan and the computation time should be taken into account.

The benefit of a heuristic method is less time computing. In case of a small size system, it may not be evident. However, in case of a large system or the addition of some security constraints, it can be obviously seen. Moreover in the multistage planning, the problem must be larger than in the case of a single stage planning. Therefore, the computation time should be the interesting issue. 


\section{REFERENCES}

[1] X. Wang and J. McDonald, Modern Power System Planning. London: McGraw-Hill, 480 pages, 1994.

[2] G. Latorre, R. D. Cruz, J. M. Areiza, and A. Villegas, "Classification of publications and models on transmission expansion planning," Power Systems, IEEE Transactions on, vol. 18, pp. 938-946, 2003.

[3] E. J. de Oliveira, I. C. da Silva, Jr., J. L. R. Pereira, and S. Carneiro, Jr., "Transmission system expansion planning using a sigmoid function to handle integer investment variables," Power Systems, IEEE Transactions on, vol. 20, pp. 1616-1621, 2005.

[4] M. V. F. Pereira and L. M. V. G. Pinto, "Application of sensitivity analysis of load supplying capability to interactive transmission expansion planning," Power Apparatus and Systems, IEEE Transactions on, vol. PAS-104, pp. 381-389, 1985.

[5] S. G. Nash and A. Sofer, Linear and Nonlinear Programming. New York: McGraw-Hill Science/Engineering/Math, 692 pages, 1996.

[6] J. Grainger and S. J. William, Power System Analysis. New York: McGraw-Hill Science/Engineering/Math, 784 pages, 1994.

[7] R. Romero, A. Monticelli, A. Garcia, and S. Haffner, "Test systems and mathematical models for transmission network expansion planning," IEE Proceedings - Generation, Transmission and Distribution, vol. 149, pp. 27-36, 2002.

[8] I. de J Silva, M. J. Rider, R. Romero, A. V. Garcia, and C. A. Murari, "Transmission network expansion planning with security constraints," Generation, Transmission and Distribution, IEEE Proceedings-, vol. 152, pp. 828-836, 2005. 